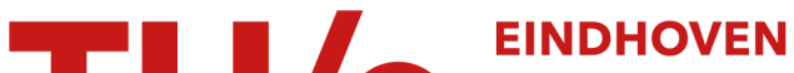 UNIVERSITY OF TECHNOLOGY
}

\section{Dynamic heterogeneity in hydrogen-bonded polymers}

\section{Citation for published version (APA):}

Muresan, A. S., Dubbeldam, J. L. A., Kautz, H., Monkenbusch, M., Sijbesma, R. P., Schoot, van der, P. P. A. M., \& Jeu, de, W. H. (2006). Dynamic heterogeneity in hydrogen-bonded polymers. Physical Review E - Statistical, Nonlinear, and Soft Matter Physics, 74(3), 031804-1/7. [031804]. https://doi.org/10.1103/PhysRevE.74.031804

DOI:

10.1103/PhysRevE.74.031804

Document status and date:

Published: 01/01/2006

\section{Document Version:}

Publisher's PDF, also known as Version of Record (includes final page, issue and volume numbers)

\section{Please check the document version of this publication:}

- A submitted manuscript is the version of the article upon submission and before peer-review. There can be important differences between the submitted version and the official published version of record. People interested in the research are advised to contact the author for the final version of the publication, or visit the $\mathrm{DOI}$ to the publisher's website.

- The final author version and the galley proof are versions of the publication after peer review.

- The final published version features the final layout of the paper including the volume, issue and page numbers.

Link to publication

\section{General rights}

Copyright and moral rights for the publications made accessible in the public portal are retained by the authors and/or other copyright owners and it is a condition of accessing publications that users recognise and abide by the legal requirements associated with these rights.

- Users may download and print one copy of any publication from the public portal for the purpose of private study or research.

- You may not further distribute the material or use it for any profit-making activity or commercial gain

- You may freely distribute the URL identifying the publication in the public portal.

If the publication is distributed under the terms of Article 25fa of the Dutch Copyright Act, indicated by the "Taverne" license above, please follow below link for the End User Agreement:

www.tue.nl/taverne

Take down policy

If you believe that this document breaches copyright please contact us at:

openaccess@tue.nl

providing details and we will investigate your claim. 


\title{
Dynamic heterogeneity in hydrogen-bonded polymers
}

\author{
Adrian S. Muresan, ${ }^{1}$ Johan L. A. Dubbeldam, ${ }^{2}$ Holger Kautz, ${ }^{3}$ Michael Monkenbusch, ${ }^{4}$ Rint P. Sijbesma, ${ }^{3}$ \\ Paul van der Schoot, ${ }^{2}$ and Wim H. de Jeu ${ }^{1}$ \\ ${ }^{1}$ FOM Institute for Atomic and Molecular Physics, Kruislaan 407, 1098 SJ Amsterdam, The Netherlands \\ ${ }^{2}$ Department of Applied Physics, Eindhoven University of Technology, P.O. Box 513, 5600 MB Eindhoven, The Netherlands \\ ${ }^{3}$ Laboratory of Macromolecular and Organic Chemistry, Eindhoven University of Technology, P.O. Box 513, 5600 MB Eindhoven, \\ The Netherlands \\ ${ }^{4}$ Institut für Festkörperforschung, Forschungszentrum Jülich, D-52425 Jülich, Germany
}

(Received 15 May 2006; published 21 September 2006)

\begin{abstract}
We report on neutron spin echo experiments on hydrogen-bonded polymers and compare the experimentally found dynamical structure factor with theoretical predictions. Surprisingly, we find that in the melt phase the expected scaling of the Rouse dynamics is not satisfied. We propose an explanation based upon the large spatial volume occupied by the connecting groups. When the effects of these bulky groups on the local friction are taken into account, the usual scaling behavior is restored.
\end{abstract}

DOI: 10.1103/PhysRevE.74.031804

PACS number(s): 82.35.Lr, 61.12.Ex, 83.80.Sg

\section{INTRODUCTION}

In systems of strongly overlapping long-chain molecules a temporary network builds up through topological entanglements between the polymer chains. The scale of these entanglements has been determined to be of the order of 4-10 nm, intermediate between the two natural lengths of polymer chains, i.e., the segment length (approximately $0.5 \mathrm{~nm}$ ) and the end-to-end distance (typically about $100 \mathrm{~nm}$ ). With these length scales different time scales can be associated. For short times, corresponding to scales up to a few times the entanglement distance, the chain segments do not strongly feel the presence of the topological constraints. The dynamics is then entropy dominated and can be associated with the so-called Rouse and breathing modes of the polymer within the confining tube formed as a result of the entanglement with its neighbors. This restricts large-scale diffusion normal to the contour of the chain.

In the standard Rouse model a melt of linear polymers is treated as a collection of Gaussian chains of length $N$ [1]. The force on a segment $n=1, \ldots, N$ of a test chain is given by the net entropic force imposed by its direct neighbors, i.e.,

$$
-k \frac{\partial^{2} \mathbf{R}(n, t)}{\partial n^{2}},
$$

in which $k=3 k_{B} T / l^{2}$ is the force constant with $l$ representing the root-mean-square size of an elementary polymer segment while $\mathbf{R}(n, t)$ is the position vector of segment $n$ at time $t$. The drag force on such a subchain segment consisting typically of 5-10 monomer units, comes from frictional drag against the background, for which long-range hydrodynamic effects may be ignored. This force equals $\zeta \partial \mathbf{R} / \partial t$ in which $\zeta$ is a drag coefficient per segment. A random force $\mathbf{f}(n, t)$ also acts on each subchain segment with a correlation time much shorter than those associated with the polymer dynamics. The correlation function $\left\langle[\mathbf{R}(n, t)-\mathbf{R}(n, 0)]^{2}\right\rangle$ describes the diffusive motion of an arbitrary segment $n$. In the Rouse model, valid for times much smaller than the reptation time, it obeys

$$
\left\langle[\mathbf{R}(n, t)-\mathbf{R}(n, 0)]^{2}\right\rangle=2 l^{2}\left(\frac{3 k_{B} T t}{\pi \zeta l^{2}}\right)^{1 / 2},
$$

at least for times smaller than the Rouse time and for segments $n$ not too close to the chain ends. The essential information about the segmental dynamics is given by the subdiffusive time dependence of the correlation function caused by the chain connectivity.

The subdiffusive segmental dynamics is well established experimentally [2], but is expected to change for hydrogenbonded polymers, at least in principle. These materials belong to the class of equilibrium polymers, i.e., linear polymeric chains that continually break and recombine, implying a coupling between reaction kinetics and polymer dynamics $[3,4]$. In this paper we consider the segmental dynamics of such polymers formed between strongly directional quadruple hydrogen-bonded dimers that in the melt potentially produce chains with a high degree of polymerization $[5,6]$. At room temperature in the solvent toluene the lifetime of the hydrogen bonds is of the order of $0.3-3 \mathrm{~s}$ [6]. Since the associated dynamics is expected to scale with viscosity, this provides a lower limit for their lifetime in a polymeric matrix. Hence, we do not expect the hydrogen-bonding kinetics to impact on the segmental motion if probed by means of neutron spin echo measurements (NSE), the reason being the large separation of the relevant time scales. However, somewhat unexpectedly we do observe a clear effect of friction heterogeneity along the backbone of the self-assembled chains. This friction heterogeneity stems from the hydrogenbonding connector groups, which in our case are quite bulky in comparison to the hydrocarbon spacers that link pairs of them in the basic molecular building blocks. We will show that incorporation of this heterogeneity into the Rouse model allows for a qualitative explanation of the experimental observations. In addition various factors will be discussed that might contribute to an improved quantitative agreement.

The paper is organized as follows. In Sec. II the hydrogen-bonded materials are described including the types of deuteration used for labeling. In addition, relevant aspects of the NSE technique are summarized. Section III presents a 


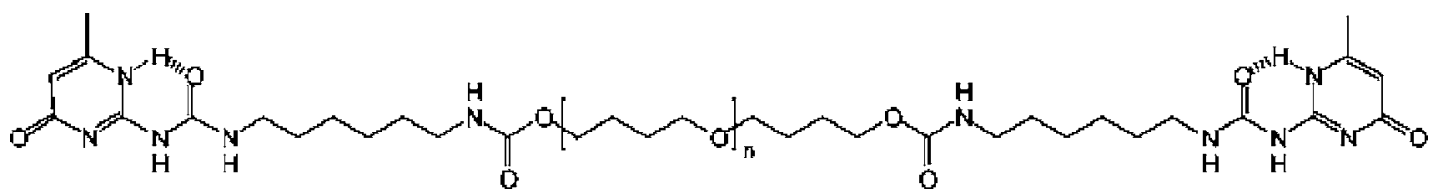

FIG. 1. Chemical structure of the molecular building block of the system used in the experiments. The two Upy groups at the ends of the elementary chain pictured are able to engage in four hydrogen bonds each, producing larger supramolecular hydrogen-bonded polymers.

theoretical discussion of the potential influence of bulky hydrogen-bonding connector groups on the dynamic structure factor. In Sec. IV the experimental results are presented and discussed in the framework of the theory. The conclusions are summarized in Sec. V.

\section{EXPERIMENT}

\section{A. Samples and labeling}

The oligomeric molecular building blocks of the system studied consisted of units of 30-60 covalently bonded tetrahydrofuran (THF) monomers with quadruple hydrogenbonding ureidopyrimidinone (UPy) groups attached at each end (see Fig. 1). We will call such a supramolecular building block an elementary chain. The hydrogen-bonding groups allow for the creation of extended hydrogen-bonded polymers [5-7]. The oligomers were prepared via the cationic polymerization of tetrahydrofuran, tetrahydrofuran- $d 8$ or the appropriate 85:15 mixture of these monomers. The procedure reported by Goethals and co-workers was followed [8], using trifluoromethanesulfonic anhydride as the initiator and 2,6di-tert-butyl pyridine as a base, while the polymerization was terminated after $12 \mathrm{~min}$ by addition of hexamethylene tetramine. The degree of polymerization of the oligomers varied from 30 to 86 due to varying initiator efficiency. Functionalization with 2-(6-isocyanatohexylaminocarbonylamino)-6methyl-4[1H]pyrimidinone was performed analogously to a previously reported procedure [9].

The total length of the self-assembled polymer is determined by the supramolecular degree of polymerization, i.e., the number of elementary units in a chain. In principle, depending on the concentration of hydrogen bonding groups and their dimerization constant, very high degrees of polymerization $(D P \gg 100)$ can be obtained [5]. In practice, the $D P$ is limited by the presence of monofunctional end groups in the system, which act as chain stoppers. The estimated lower limit of functionalization is 1.9 per chain, which would imply $D P>20$. However, as the Rouse length scale is shorter than the length of the elementary unit, the supramolecular $D P$ should be irrelevant save for end effects. The time scale of equilibration of the self-assembled system compared to the relevant time scale of a NSE measurement is such that the latter sees an effective polydispersity which is the most probable or Flory distribution with $M_{w} / M_{n} \approx 2$.

We have studied the material with two different types of labeling, A and B, illustrated in Figs. 2(a) and 2(b). In labeling $A$ the elementary chains are identical and have $15 \%$ hydrogenated monomers distributed at random in a matrix of deuterated monomers. The molar mass $M_{n}=5.8 \mathrm{~kg} / \mathrm{mol}$ of an elementary chain corresponds to $\approx 74$ monomers. In la- beling $\mathrm{B}$ we have a mixture of $15 \%$ hydrogenated elementary chains with a molar mass of $M_{n}=2.5 \mathrm{~kg} / \mathrm{mol}(\approx 30$ monomers) and $85 \%$ deuterated elementary chains with a molar mass of $M_{n}=7.4 \mathrm{~kg} / \mathrm{mol}$ corresponding to $\approx 86$ monomers. Due to the breaking and recombination of hydrogen bonds, the spatial distribution of the hydrogenated elementary chains will be random. The two types of labeling A and B are qualitatively similar, the only difference being the size of the randomly placed hydrogenated unit: a single monomer in case $\mathrm{A}$, a full elementary chain $\approx 30$ monomers in case $\mathrm{B}$.

\section{B. Neutron spin echo measurements}

The method of choice to measure the short time dynamics of polymer chains is neutron spin echo. With the NSE method one measures the final polarization of a neutron beam as a function of the initial polarization as given by [2]

$$
P_{f}=P_{i} \frac{\int_{-\infty}^{\infty} S(q, \omega) \cos (\omega t) d \omega}{\int_{-\infty}^{\infty} S(q, \omega) d \omega}
$$

in which $S(q, \omega)$ is the dynamic structure factor of the system that depends on the momentum transfer $q$ and the frequency $\omega$. Hence, NSE measures the $q$ and $t$ dependent correlation function of the studied system.

Deuterium scatters mainly coherently, while hydrogen scatters incoherently. If a polymer sample is labelled by a majority of deuterated monomers and in this deuterated matrix one inserts a small percentage of hydrogenated monomers at random sites, then the signal stems from the contrast difference between the deuterated and hydrogenated parts.

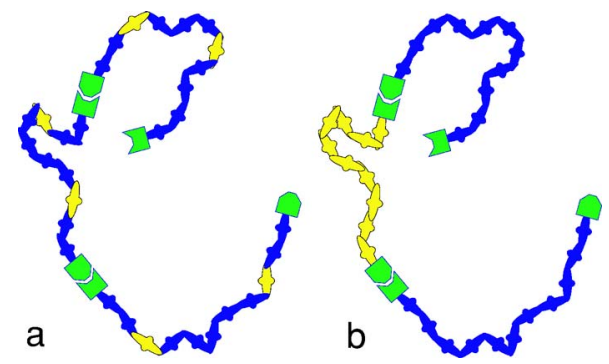

FIG. 2. (Color online) Schematic model of the labeled suprapolymeric systems A (a) and B (b). The deuterated blue and hydrogenated yellow monomers are covalently bound while the green blocks represent the quadruple hydrogen bonding Upy groups. The hydrogenated monomers represent around $15 \%$ of the total number of monomers in system $\mathrm{A}$ and the hydrogenated chains represent around $15 \%$ of the total number of chains in system B. 
By measuring the time correlation of the intensity scattered by the deuterated monomers we are actually probing the dynamics of that part of the system that does not scatter coherently (the holes), i.e., the $15 \%$ hydrogenated monomers. As a result the coherent NSE signal in a sample labeled as $15 \%$ hydrogenated monomers in $85 \%$ deuterated monomers will give the self-correlation of the hydrogenated monomers. Hence the monomer self-correlation function can be measured, which is within the Gaussian approximation, e.g., for a Rouse chain, given by [1]

$$
S(q, t)=\frac{1}{N} \sum_{k=1}^{N} \exp \left\{-\left(q^{2} / 6\right)\left\langle\left[\mathbf{R}_{k}(t)-\mathbf{R}_{k}(0)\right]^{2}\right\rangle\right\} .
$$

The monomer self-correlation of Eq. (4), also called dynamic structure factor, can in principle also be obtained by measuring the incoherent NSE signal in a fully hydrogenated sample. The time scale for taking such an incoherent NSE scan is a few weeks, which is impractical and the reason why we measured coherently. In fact the NSE experiments we have performed measure $S_{\text {coh }}(Q, t)-\frac{1}{3} S_{\text {inc }}(Q, t)$, which in the $q$-range investigated is dominated by the coherent scattering (see below).

Introducing Eq. (2) into Eq. (4) we obtain for the dynamic structure factor

$$
\frac{S(q, t)}{S(q, 0)}=\exp \left\{-(2 / \sqrt{\pi})\left[\Omega_{R}(q) t\right]^{1 / 2}\right\}
$$

with the Rouse kinetics determined by the rate

$$
\Omega_{R}(q)=\frac{1}{12} k_{B} T\left(l^{2} / \zeta\right) q^{4} .
$$

Hence, from these relations we expect

$$
S(q, t) \sim \exp \left(-\alpha q^{2} \sqrt{t}\right)
$$

to hold. The segmental friction coefficient-the quantity to be determined - can be calculated from the coefficient of the exponential of decay $S\left(q^{2} \sqrt{t}\right)$ as

$$
\frac{\zeta}{l^{2}}=\frac{k_{B} T}{3 \pi \alpha^{2}} .
$$

We measured the ratio $S(q, t) / S(q, 0)$ of the polymer melts using the FRJ2-NSE spectrometer at the DIDO reactor of the FZ-Jülich $[10,11]$. The neutron wavelength band used was centered at $\lambda=0.8 \mathrm{~nm}$ with a width [full width at half maximum (FWHM)] of 10\%; the beam had a cross section of $30 \times 30 \mathrm{~mm}^{2}$. Scattered neutrons were collected using a two-dimensional detector covering an effective detection area of $2^{\circ}$ radius. Data analysis yielded $S(q, t) / S(q, 0)$ for four different $q$-values per detector setting. Data were collected for scattering arm settings corresponding to $q=0.5$, $0.8,1.0,1.4,1.7,2.0,2.4$, and $2.8 \mathrm{~nm}^{-1}$ at the detector center and for Fourier times $0.15<t<22 \mathrm{~ns}$. The samples were held in $4 \mathrm{~mm}$ thick niobium cuvettes at a temperature of $353 \mathrm{~K}$. The sample with labeling B was also measured at 333 and $373 \mathrm{~K}$. The results indicated a small increase $(10 \%-$ $20 \%$ ) of the coherent scattering with temperature. As a back-
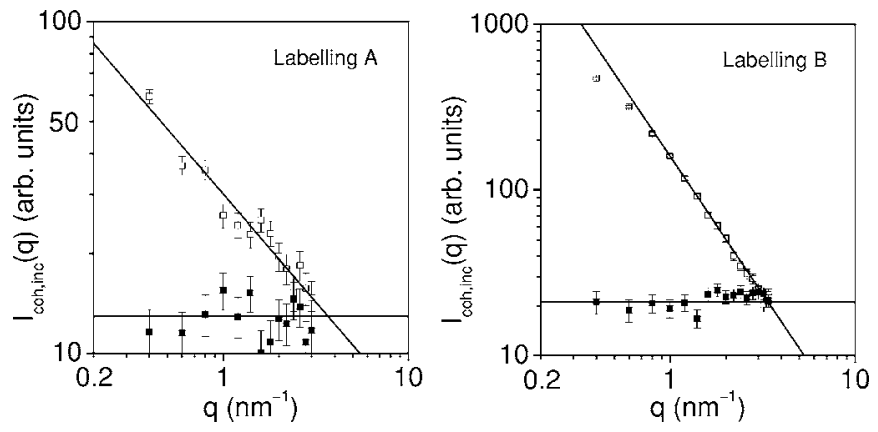

FIG. 3. Coherent (open squares) and incoherent (filled squares) contributions to the total scattered intensity for labeling A and B at $353 \mathrm{~K}$. The slopes of the coherent intensity as indicated follow $q^{-0.66}$ (labeling A) and $q^{-1.68}$ (labeling B), respectively.

ground a fully deuterated polymer sample was measured and subsequently subtracted during the data treatment to yield $S(q, t) / S(q, 0)$.

Using the implicit polarization analysis, we checked the relative contributions from coherent and incoherent scattering. The results shown in Fig. 3 indicate that up to about $q=0.24 \mathrm{~nm}^{-1}$ the coherent scattering dominates. Only at the end of the $q$-range used in the dynamic NSE experiments the coherent intensity starts to drop below the incoherent level. Because of limited beamtime the experiments on the samples with labeling A are not of the same high quality as those for labeling B.

\section{THEORY}

To evaluate any potential influence of the bulky connecting UPy groups on the dynamic structure function $S(q, t)$, we present in this section a Rouse model for a multichain built up from smaller subchains with UPy groups at the ends. The total chain can thus be viewed as a copolymer consisting of two species: THF monomers and connector UPy groups that are periodically arranged. In our model we take the UPy groups to be located at sites $0, M, 2 M, \ldots, N$, all the other sites are occupied by monomers. We presume that the UPy connector groups have friction coefficient $\zeta_{G}$ that is considerably larger than $\zeta_{0}$, the friction coefficient of an elementary Kuhn segment ( $\approx 4$ monomers) of the chain. A sketch of the model, showing arbitrarily seven subchains which together constitute the polymer that we examine, is presented in Fig. 4.

The equations of motion for the total Rouse chain read [1]

$$
\dot{\mathbf{R}}_{i}=\left(\mathbf{R}_{i+1}+\mathbf{R}_{i-1}-2 \mathbf{R}_{i}\right) \frac{3 k_{B} T}{\zeta_{0} l^{2}}+\frac{\mathbf{f}_{i}(t)}{\zeta_{0}}
$$

for $i=1, \ldots, M-1, M+1, \ldots, 2 M-1,2 M+1, \ldots N-1$. In Eq. (9), $\mathbf{R}_{i}$ denotes the coordinate of the $i$ th Kuhn segment and differentiation with respect to time is designated by a dot. The two connecting UPy connector groups at $i=0$ and $i=N$, and $j=M, 2 M, \ldots$, obey the relations 


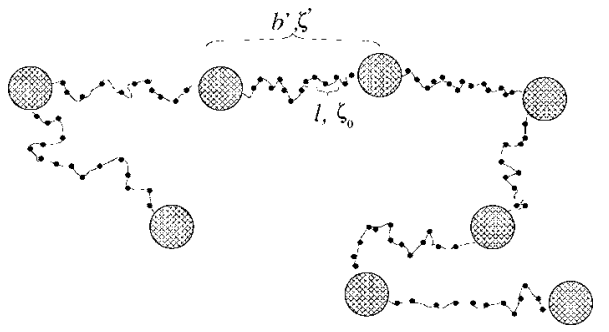

FIG. 4. Theoretical model of a Rouse chain built up from smaller chains with two functional groups that are extended in space and represented by larger beads. The latter beads have a larger friction than the smaller ones representing the monomers, which destroy the invariance of the Rouse chain under scaling transformations.

$$
\begin{gathered}
\dot{\mathbf{R}}_{0}=\left(\mathbf{R}_{1}-\mathbf{R}_{\mathbf{0}}\right) \frac{3 k_{B} T}{\zeta_{G} l^{2}}+\frac{\mathbf{f}_{0}(t)}{\zeta_{G}}, \\
\dot{\mathbf{R}}_{N}=\left(\mathbf{R}_{N-1}-\mathbf{R}_{\mathbf{N}}\right) \frac{3 k_{B} T}{\zeta_{G} l^{2}}+\frac{\mathbf{f}_{N}(t)}{\zeta_{G}}, \\
\dot{\mathbf{R}}_{j}=\left(\mathbf{R}_{j+1}+\mathbf{R}_{j-1}-2 \mathbf{R}_{j}\right) \frac{3 k_{B} T}{\zeta_{G} l^{2}}+\frac{\mathbf{f}_{j}(t)}{\zeta_{G}},
\end{gathered}
$$

where $\mathbf{f}_{i}(t)$ represents the Brownian force exerted on the molecules and $l$ is the length of a Kuhn segment; see also Fig. 4. The Brownian forces are assumed to be Gaussian with zero mean and delta correlated in time according to $\left\langle f_{i}^{\alpha}\left(t_{1}\right) f_{j}^{\beta}\left(t_{2}\right)\right\rangle=2 k_{B} T \zeta_{0, G} \delta_{i j} \delta_{\alpha \beta} \delta\left(t_{1}-t_{2}\right)$, where $\zeta_{0, G}$ equals $\zeta_{0}$ for the Kuhn monomers and $\zeta_{G}$ for the connector groups. We introduce the ratio of friction constants $r=\zeta_{0} / \zeta_{G}$, which is smaller than unity in our case of spatially extended connectors, and reduces to $r=1$ for the homopolymer Rouse chain. Time is made dimensionless by scaling it to $\tau_{0}=\zeta_{0} l^{2} /\left(3 k_{B} T\right)$, the time for a single Kuhn segment to relax. Because the Rouse model is not valid on length scales smaller than the Kuhn length, the relevant time regime is $t>\tau_{0}$. The system of differential equations (9) and (10) can be solved analytically for special values of $r$. For that purpose it is convenient to rewrite these equations in matrix form,

$$
\dot{\mathbf{R}}=-\stackrel{\leftrightarrow}{A} \cdot \mathbf{R}+\mathbf{g}(t)
$$

where the matrix $\overleftrightarrow{A}$ is defined by

$$
\left(\begin{array}{cccccc}
r & -r & 0 & 0 & 0 & 0 \\
-1 & 2 & -1 & . & . & . \\
0 & -1 & 2 & -1 & 0 & 0 \\
0 & 0 & \cdot & \cdot & . & 0 \\
\cdot & \cdot & \cdot & -1 & 2 & -1 \\
0 & 0 & 0 & 0 & -r & r
\end{array}\right) .
$$

In the matrix $\overleftrightarrow{A}$ the entries of the $M, 2 M, \ldots$, th row are $2 r$ at the diagonal and $-r$ at the entry left and right from this diagonal element. The components of $\mathbf{g}(t)$ satisfy $\left\langle g_{i}^{\alpha}\left(t_{1}\right) g_{j}^{\beta}\left(t_{2}\right)\right\rangle=\left(2 l^{2} / 3\right) \delta_{i j} \delta_{\alpha \beta} \delta\left(t_{1}-t_{2}\right)$, for $0<i<N$ except when $i$ is a multiple of $M, i=0$ or $i=N$. In those cases the delta function must be multiplied by $r$.

Solving Eq. (11) actually boils down to calculating the eigenvalues and eigenvectors of matrix $\stackrel{\leftrightarrow}{A}$. For this task one has in general to resort to numerical computations. We performed the numerical determination of the eigenvalues using a cyclic Jacobi routine. In a few limiting cases exact results may be obtained. Of course, the case $M=2$, in which the monomers and the connector groups are alternating, can be solved easily. More interestingly, for sufficiently high frequencies, we can find an asymptotically correct expression for the eigenvalues $\lambda_{k}$. In this case the monomers in the chain will only notice their local environment and the whole chain can be approximated by a single subchain consisting of M-1 monomers and two connector groups at the chain ends. We find that the eigenvalues for a chain with only two bulky groups at the ends can be found by solving the transcendental equations for $\theta_{k}$,

$$
\begin{gathered}
\tan \left(\theta_{k} / 2\right)\left(1-\frac{2}{r}\right)=\tan \left(\theta_{k} M / 2\right), \\
\tan \left(\theta_{k} / 2\right)\left(1-\frac{2}{r}\right)=-\left[\tan \left(\theta_{k} M / 2\right)\right]^{-1},
\end{gathered}
$$

where we introduced $\theta_{k}$, which is related to the eigenvalues $\lambda_{k}$ by $\lambda_{k}=2\left(1-\cos \theta_{k}\right)$. Note that taking $r=1$ leads to the correct Rouse eigenvalues $\lambda_{k}=\sin [\pi k /(M+1)]$, with $k=0,1, \ldots M$. The limiting cases $r=0$ and $r=\infty$ can be viewed as special cases which can easily be treated as Rouse chains with fixed or loose endpoints, respectively.

To obtain the mean square displacement of a polymer segment $\left\langle\left[\mathbf{R}_{n}(t)-\mathbf{R}_{n}(0)\right]^{2}\right\rangle$ and thus the structure factor $S(q, t)$, we calculate the eigenvalues and eigenvectors of $\overleftrightarrow{A}$ and find

$$
\begin{aligned}
\left\langle\left[\mathbf{R}_{i}(t)-\mathbf{R}_{i}(0)\right]^{2}\right\rangle= & 2 l^{2} \sum_{j, k, l=0}^{N} S_{i j} S_{i l}\left(S^{-1}\right)_{j k}\left(S^{-1}\right)_{l k} \\
& \times \frac{\left(1-e^{-\left(\lambda_{j}+\lambda_{l}\right) t}\right)}{\lambda_{j}+\lambda_{l}}\left(1+(r-1) \sum_{n=0}^{N} \delta_{k, n M}\right) .
\end{aligned}
$$

The matrix $\overleftrightarrow{S}$ contains the eigenvectors associated with the eigenvalues $\lambda_{k}$ of $\overleftrightarrow{A}$, and $\overleftrightarrow{S}^{-1}$ is the inverse of $\overleftrightarrow{S}$.

In Fig. 5, we show the structure function $S(q, t)$ as a function of time for a fixed value of $q=l^{-1}$. We took a full polymer chain with $N=100$ monomers divided in five elementary chains of 20 monomers each $(M=20)$, corresponding to the experimental situation if we identify a Kuhn segment of length $l$ with about four monomers. The bulky connector groups are assumed to have a friction $\zeta_{G}$ that is 100 times the monomer friction $\zeta_{0}$, corresponding to $r=0.01$. From Fig. 5 it is clear that the Rouse expression Eq. (5) for $S(q, t)$ is in nearly perfect agreement with the numerical result at the time scales we are interested in. Deviations at short time scales arise, as the stretched exponential expression for $S(q, t)$ is not valid anymore for $t / \tau_{0} \ll 1[1]$. This situation 


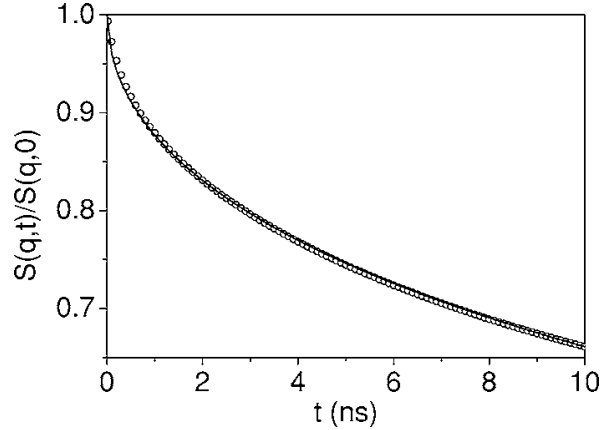

FIG. 5. The structure factor $S(q, t)$ as a function of time for parameter values $N=100, M=20, r=0.01$. When fixing the time scale $\tau_{0}$ described in the main text to ns, we presumed the Kuhn length of a segment to be about $1 \mathrm{~nm}$ and the friction coefficient $\zeta_{0} \simeq 10^{-10} \mathrm{Ns} / \mathrm{m}$. As $r=0.01$ the connector groups have a 100 times larger friction. The numerical computation (circles) is seen to agree well with the Rouse result for homopolymers (solid curve) represented by $\exp (-0.131 \sqrt{t})$.

applies to labeling $\mathrm{A}$ in which the majority of the monomers are well separated from the connector groups, and do not feel much of the increased friction. As a result Eq. (5) is still expected to hold, even though we have a dynamically heterogeneous chain.

To describe labeling B we consider the effects of taking a larger Kuhn length $l^{\prime}$, equal to the size of an elementary chain with two connector groups. The transition from labeling A to labeling B amounts to performing the transformation $l \rightarrow l^{\prime}$ for the Kuhn length, and $\zeta_{0} \rightarrow \zeta^{\prime}$ for the friction. The values of $l$ and $l^{\prime}$ are related by $l^{\prime 2}=l^{2} M$, which is true for any Rouse chain. However, the expression $\zeta^{\prime}=M \zeta_{0}$ that is valid for ordinary chains, that is, for the case $r=1$, no longer holds. The friction, which is presumed additive in the freedraining (Rouse) limit, has contributions from both the connector groups coefficient $\zeta_{G}$ and the small monomers with a friction coefficient $\zeta_{0}$. If we add the friction of the monomers without and with connector groups, we obtain $\zeta^{\prime}$ $=0.15 \zeta_{0}(M-2+2 / r)+0.85 \zeta_{0}[M-2+2 /(3 r)]$, in which we have taken into account that $15 \%$ of the chains is deuterated having length $M$ and $85 \%$ is hydrogenated having a three times longer length. Therefore on average only $2 / 3$ of a connector group is present in the hydrogenated chain of length $M$. Adding the two terms gives $\zeta^{\prime}=\zeta_{0}(M-2+0.87 / r)$, which is dominated by the friction of the bulky groups. This means that, contrary to the Rouse result, $l^{2} / \zeta$ and therefore $S(q, t)$ changes as a result of the scaling transformation associated with the relabeling $\mathrm{A} \rightarrow \mathrm{B}$. For values $M=20, r=0.01$, we find that $l^{2} \zeta^{\prime} /\left(l^{\prime 2} \zeta_{0}\right)=5.2$, which illustrates that the large connecting groups make the relaxation slower for all values of $q$.

\section{RESULTS AND DISCUSSION}

Figure 6 shows the correlation functions for samples A and $\mathrm{B}$ as obtained by NSE for a range of $q$-values at $T=353 \mathrm{~K}$. The data is well fitted by the Rouse model, i.e., by a function of the form of Eq. (7) at each $q$. As anticipated in
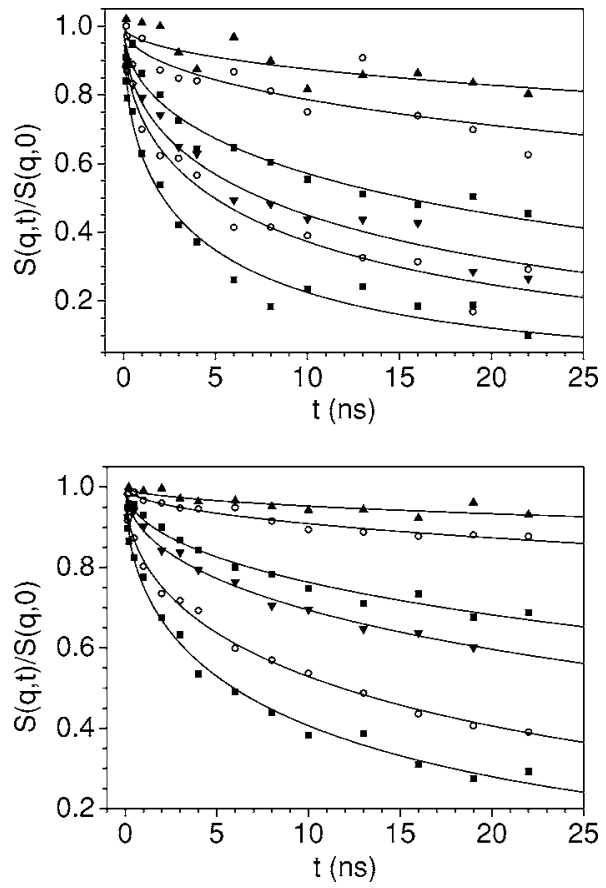

FIG. 6. NSE correlation functions of sample A (a) and B (b) for $T=353 \mathrm{~K}$ at (from top to bottom) $q=0.8,1.0,1.4,1.7,2.0$, and $2.4 \mathrm{~nm}^{-1}$. Fits of the correlation functions to Eq. (14) (with $y=1 / 2$ ) are also shown.

the introduction, at the time scale of the experiments no direct effect of the hydrogen-bonding kinetics shows up. We have also fitted the correlation functions at different $q$-values with a function of the form

$$
\frac{S(q, t)}{S(q, 0)}=\exp \left(-\beta t^{y}\right)
$$

to check if there is a reason for a different time dependence than the power $1 / 2$ of $t$ in the exponent. Fitting all values of $q$, we obtained $y_{A}=0.57 \pm 0.13$ and $y_{B}=0.507 \pm 0.047$. Hence we adhere to a fixed exponent of 0.5 . Figure 7 shows a logarithmic plot of the correlation functions taken at $T=353 \mathrm{~K}$. For both types of labeling the decay functions can be well fitted by Eq. (7). The decay is slower for the system with

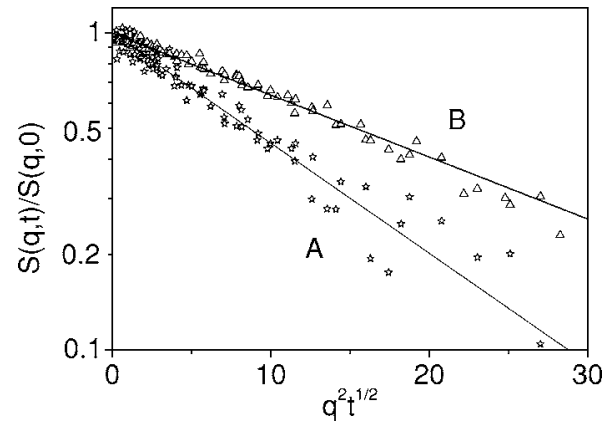

FIG. 7. Logarithmic plot of the correlation function for the system with labeling A and $\mathrm{B}$, respectively, at $T=353 \mathrm{~K}$. The data is plotted as a function of $q^{2} \sqrt{t}$, with $q$ represented in $\mathrm{nm}^{-1}$ and $t$ in ns. The solid lines show fits to the exponential decay function Eq. (7). 
labeling B as compared to labeling A. The calculated segmental friction coefficients are

$$
\begin{gathered}
\zeta / l^{2}=0.84 \times 10^{8} \mathrm{Ns} / \mathrm{m}^{3} \text { for labeling A, } \\
\zeta^{\prime} / l^{\prime 2}=2.55 \times 10^{8} \mathrm{Ns} / \mathrm{m}^{3} \text { for labeling B. }
\end{gathered}
$$

The standard Rouse model for dynamically homogeneous chains predicts that the two segmental friction coefficients are the same, therefore the two slopes shown in Fig. 7 should be the same because of dynamic scale invariance. We attribute the observed slowing down for labeling B to the dynamic heterogeneity of the system discussed theoretically in Sec. III. For the present experimental situation this can be understood as follows. For labeling A, the labeled monomers are distributed along the elementary chain. Only small monomers close to the large connecting UPy groups will feel their effect and slow down. The dynamics of the majority of small monomers further away from the bulky groups will not feel the slowing-down effect. In contrast, for the labeling B, due to the fact that the full elementary chain is labeled, the slowing-down effect by the bulky connectors cannot be avoided.

More quantitatively, we put forward that the usual Rouse scaling for homopolymers does not hold anymore for labeling B. The new labeling B corresponds to a scaling transformation in which the Kuhn length $l \rightarrow l^{\prime}$ and the friction $\zeta \rightarrow \zeta^{\prime}$. From the number of Kuhn segments in an elementary chain of length $M$, we have the relation $l^{\prime 2}=l^{2} M$. The corresponding friction of a segment containing $M$ monomers labeled A is $\zeta^{\prime}=\zeta_{0}(M-2+0.87 / r)$. If we take $M \approx 20$ and use the ratio $r$ as a fit parameter, we find good agreement with the experimental result $l^{2} \zeta^{\prime} /\left(l^{2} \zeta_{0}\right)=3$ for $r=0.021$. From this result the effective friction of the UPy groups is about 50 times larger than that of the small monomers.

From molecular modeling we calculate a volume ratio of UPy dimer to a small monomer of about six. For a simple Debye-type model for which viscosity scales linearly to a relevant size, this number would give an upper bound to the friction ratio, leading to a significant quantitative discrepancy with the experiment. What could be the origin of such a huge difference in mobilities of the two kinds of elementary unit of these self-assembled copolymers? First of all, in our highly idealized model we did allow for a heterogeneity in the friction constant of the two units but not in the spring constants. While it is gratifying that the model does qualitatively explain our measurements, this means that any spring constant heterogeneity could impact on the ratio of the friction constants that we extract from the experiments, i.e., they are essentially effective ones. Alternatively, the friction constants, and in particular one of the larger UPy groups, may in fact not be that of a free unit even if this effect were negligible. Hence we should consider potential sources of friction constant renormalization.

Rheological data of related hydrogen-bonded polymers show a low frequency plateau in the dynamic shear modulus $G^{\prime}[9,12]$. This indicates that a fraction of the dimerized UPy groups forms lateral stacks, which would affect the friction of these units. Though such an effect is less likely for the present system because of different subchains, it cannot be excluded at this stage. Such a networklike structure would further decrease the mobility of the connector groups and neighboring monomers. Then one would experimentally observe not the friction coefficient of a single connector group $\zeta_{G}$, but that of an aggregate of connector groups that would consequently be much larger.

Finally we could assume that the friction of the connecting UPy groups is actually much larger than expected if we only consider that part of the Stokes friction that scales like the reciprocal size of these units. Odijk has discussed the effect of polymer depletion on the transport of small particles in a semidilute solution [13]. For particles that are larger than the length of a Kuhn segment of the polymer but smaller than the entanglement length, the diffusion is restricted by the accessible volume. This leads to a retardation factor that brings the length scale involved effectively into an exponential. Though our situation is rather different (no semidilute solution, bulky connector group not free from the polymer chain) one could apply similar arguments assuming that the topological network of entangled chains must have a similar effect in the melt, too. In that case a factor of the order of six in the volume ratio could give a huge effect on the diffusivity/friction. At this stage we cannot decide which of the features mentioned (or combination of them) applies to the present situation. Obviously it would be of interest to investigate this issue, something we hope to do in the near future.

\section{CONCLUSIONS}

The dynamics of hydrogen-bonded polymers has been shown to be influenced by their friction heterogeneity due to the presence of small monomers in the elementary chains that are in turn connected by bulky interconnecting UPy groups carrying four hydrogen bonds. At small length scales corresponding to a few Kuhn lengths (labeling local monomers) the presence of UPy groups is not noticed and the observed relaxation is well described by the usual Rouse relaxation, following a stretched exponential. Changing the labeling to the full elementary chain is a way to look effectively at longer length scales. In contrast to ordinary homopolymeric Rouse chains, for which the scaling of friction is linear with the number of monomers and the relaxation of the structure function is independent of the labeling, now a slowing down of the relaxation is observed. This situation corresponds to a scaling transformation of the Rouse chain by increasing the Kuhn length. By incorporating the larger friction experienced by the bulky UPy groups, the observed slower relaxation can be rationalized. Various possibilities have been suggested that can account for the observation that this effect is considerably larger than anticipated on the basis of the simple model.

\section{ACKNOWLEDGMENTS}

This work is part of the research program of the Stichting voor Fundamenteel Onderzoek der Materie (FOM) which is financially supported by the Nederlandse Organisatie voor Wetenschappelijk Onderzoek (NWO). 
[1] M. Doi and S. F. Edwards, The Theory of Polymer Dynamics (Clarendon, Oxford, 1986).

[2] D. Richter, M. Monkenbusch, A. Arbe, and J. Colmenero, Adv. Polym. Sci. 174, 1 (2005).

[3] M. E. Cates, Macromolecules 20, 2289 (1987).

[4] M. E. Cates and S. J. Candau, J. Phys.: Condens. Matter 2, 6868 (1990).

[5] R. P. Sijbesma et al., Science 278, 1501 (1997).

[6] S. H. M. Söntjens, R. P. Sijbesma, M. H. P. van Genderen, and E. W. Meijer, J. Am. Chem. Soc. 122, 7487 (2000).

[7] L. Brunsveld, B. J. B. Folmer, E. W. Meijer, and R. P. Sijbesma, Chem. Rev. (Washington, D.C.) 101, 407 (2001).

[8] M. F. Dubreuil, N. G. Farcy, and E. J. Goethals, Macromol.
Rapid Commun. 20, 383 (1999).

[9] B. J. B. Folmer, R. P. Sijbesma, R. M. Versteegen, R. M. J. A. J. van der Rijt, and E. W. Meijer, Adv. Mater. (Weinheim, Ger.) 12, 874 (2000).

[10] M. Monkenbusch, R. Schatzler, and D. Richter, Nucl. Instrum. Methods Phys. Res. A 399, 301 (1997).

[11] Due to the end of operation of the FRJ2-DIDO reactor in Jülich the NSE instrument has been moved to the new research reactor FRMII in Münich in March 2006 and will be available there soon.

[12] D. J. M. van Beek, A. J. H. Spiering, G. W. M. Peters, R. P. Sijbesma, and E. W. Meijer (unpublished).

[13] T. Odijk, Biophys. J. 79, 2314 (2000). 